\title{
Synthesis of 3-substituted pyridinium salts
}

\author{
Juliusz Pernak* and Jarosław Rogoża \\ Institute of Chemical Technology and Engineering, Poznań University of Technology, 60-965 \\ Poznań, pl. Skłodowskiej-Curie 2, Poland \\ E-mail: Juliusz.Pernak@put.poznan.pl
}

(received 20 Apr 00; accepted 26 Nov 00; published on the web 04 Dec 00)

\begin{abstract}
A novel class of 3-substituted pyridinium salts have been synthesised in high yield by a convenient two-step procedure. A new synthetic pathway to 1-substituted benzimidazolium salts has been developed and the effects of the anionic component of the salts have been studied.
\end{abstract}

Keywords: Alkylations, Mannich bases, condensations, pyridinium salts, benzimidazolium salts

\section{Introduction}

Compounds containing a pyridinium moiety attached to a heterocyclic system are important in natural product chemistry ${ }^{1}$ and in organic synthesis ${ }^{2,}$. . Pyridinium salts have found use as acylating agents ${ }^{4}$, phase transfer catalysts ${ }^{5}$, biocides with a wide range of antimicrobial activity ${ }^{6}$, dyes $^{7}$ and cationic surfactants. The 1-alkylpyridinium salts, which are liquid at rt., so-called ionic liquids, are potential new solvents for synthesis and catalysis ${ }^{8}$. Several synthetic routes to pyridinium salts are known, but the most commonly used method is the Menschutkin reaction, the SN2 reaction of a pyridine derivative with an organic halide. Chloromethylalkyl ethers or sulphides are also reagents for the quaternization of the pyridine nitrogen. In these cases the reactions proceed via the SN1 mechanism ${ }^{9-11}$.

The present paper reports on a general and convenient route for the preparation of 3substituted pyridinium salts such as shown in Figure 1 by utilization of N-Mannich bases as starting materials.

The $N$-(1H-benzimidazolalkyl)-3-pyridinecarboxamides (1a and 1b) are new compounds which were obtained in a one-pot condensation reaction of benzimidazole-aldehydenicotinamide (Scheme 1). 


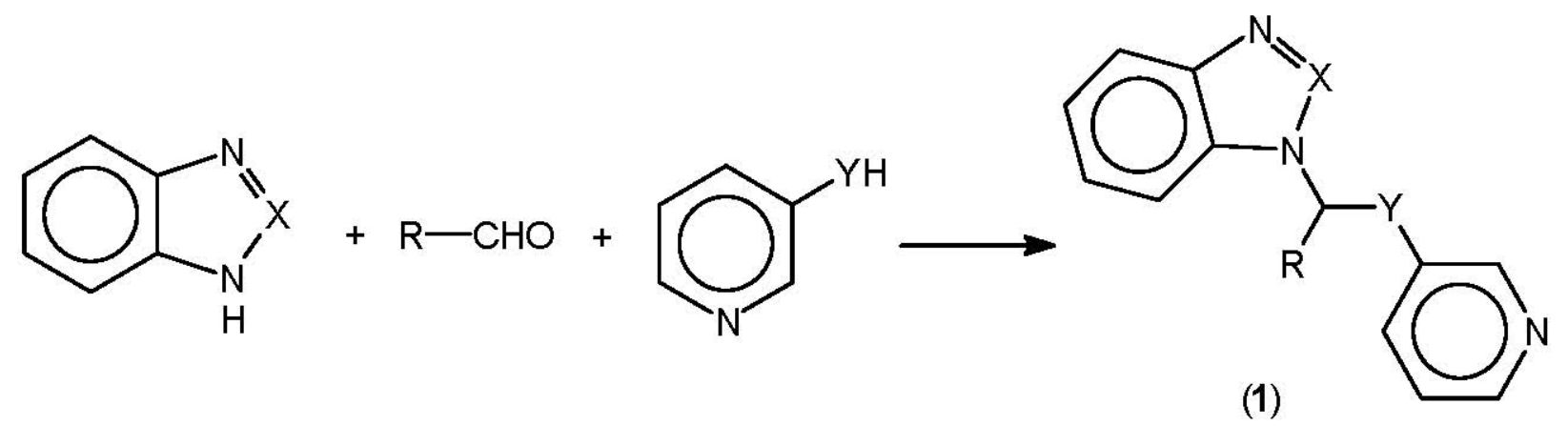

\section{Scheme 1}

The chemotypes which have been prepared along with reaction times, yields and melting points are tabulated in Table 1.

The limitation of this procedure is that only two aldehydes, formaldehyde and benzaldehyde undergo reaction with benzimidazole and nicotinamide.

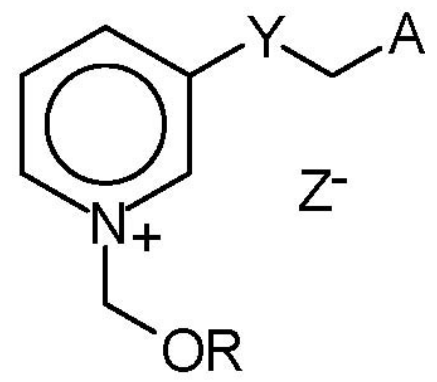

$$
\mathrm{Y}=\mathrm{CONH} \text { or } \mathrm{NH} ; \mathrm{A}=\text { azole }
$$

\section{Figure 1}

The compounds were characterised by their $1 \mathrm{H}$ and 13C NMR spectra and by elemental analyses. The NH proton for $\mathbf{1 a}$ resonated at $\delta=9.93-9.89$ as a triplet with a coupling constant in the range $5.6 \mathrm{~Hz}$. The methine protons appeared in the spectrum as a doublet at $\delta=5.83-5.81$ $(J=6 \mathrm{~Hz})$. The chemical shift of the carbonyl carbon appeared at $\delta=165.3$. As expected, the absorption peak for the characteristic $\alpha$ carbon between the benzimidazole ring and the amide fall in the region of $\delta=53-48$. Analogue 1c was prepared by treatment of 3-aminopyridine with formaldehyde and benzimidazole. 
Table 1. Condensation Products 1

\begin{tabular}{ccccccc}
\hline Product & $\mathrm{R}$ & $\mathrm{X}$ & $\mathrm{Y}$ & Time $(\mathrm{h})$ & Yield $(\%)$ & m.p. ${ }^{\mathrm{a}}\left({ }^{\circ} \mathrm{C}\right)$ \\
\hline $1 \mathrm{a}$ & $\mathrm{H}$ & $\mathrm{CH}$ & $\mathrm{CONH}$ & 48 & 90 & $218-220$ \\
$1 \mathrm{~b}$ & $\mathrm{C}_{6} \mathrm{H}_{5}$ & $\mathrm{CH}$ & $\mathrm{CONH}$ & 48 & 45 & $230-232$ \\
$1 \mathrm{c}$ & $\mathrm{H}$ & $\mathrm{CH}$ & $\mathrm{NH}$ & 5 & $80^{\mathrm{b}}$ & $160-162^{\mathrm{c}}$ \\
$1 \mathrm{~d}$ & $\mathrm{H}$ & $\mathrm{N}$ & $\mathrm{CONH}$ & 48 & 86 & $177-178^{\mathrm{d}}$ \\
$1 \mathrm{e}$ & $\mathrm{C}_{6} \mathrm{H}_{5}$ & $\mathrm{~N}$ & $\mathrm{CONH}$ & 48 & 75 & $184-186^{\mathrm{e}}$ \\
$1 \mathrm{f}$ & $\mathrm{H}$ & $\mathrm{N}$ & $\mathrm{NH}$ & 5 & $80 \mathrm{~b}$ & $153-154_{\mathrm{f}}$ \\
\hline
\end{tabular}

${ }^{a}$ Solvent for recrystallization: $\mathrm{MeOH}(\mathbf{1 a}, \mathbf{b}), \mathrm{EtOH} / \mathrm{H}_{2} \mathrm{O}(\mathbf{1} \mathbf{c}-\mathbf{f}),{ }^{\mathrm{b}}$ The same yield in microwave reactor (time $10 \mathrm{~min} ., 200^{\circ} \mathrm{C}, 850 \mathrm{~W}$ ), ${ }^{\mathrm{c}}$ Lit. ${ }^{12} \mathrm{~m} . \mathrm{p} .163-164^{\circ} \mathrm{C},{ }^{\mathrm{d}}$ Lit. ${ }^{13} \mathrm{~m} . \mathrm{p} .177-178^{\circ} \mathrm{C}$, ${ }^{\mathrm{e}}$ Now is available in Aldrich, ${ }^{\mathrm{f}}$ Lit. ${ }^{12}$ m.p. $150-151^{\circ} \mathrm{C}$

The N-Mannich reagents, N-(1 H-benzotriazol-1-ylmethyl)-3-pyridinecarboxamide (1d) and 3-(benzotriazol-1-ylmethylamino)pyridine (1f), were easily prepared by the condensation of benzotriazole-formaldehyde with the appropriate amide or amine ${ }^{14,15}$. The 3-aminopyridine reacts with formaldehyde and benzotriazole at $\mathrm{rt}$ to give monosubstituted product 1f, previously prepared by the condensation of 3-aminopyridine with 1-(hydroxymethyl)benzotriazole ${ }^{14}$.

When the condensed product $\mathbf{1 d}$ was prepared directly from nicotinamide, formaldehyde and benzotriazole, the reaction conditions required were more vigorous due to the lower nucleophilicity of nicotinamide compared to 3-aminopyridine.

Analogue 1e was prepared from pyridine-3-carboxamide-benzaldehyde-benzotriazole. The reactions of benzotriazole was carried out in high yield with the regioselective formation of the $\mathrm{N}-1$ isomer. In this condensation, azoles other than benzotriazole have been utilized (imidazole ${ }^{16}$, triazole $\left.{ }^{13}\right)$.

The quaternization of a N-Mannich bases such as benzimidazoles 1a and 1c by chloromethylalkyl ether afforded two products 2 and 3 (Scheme 2). 

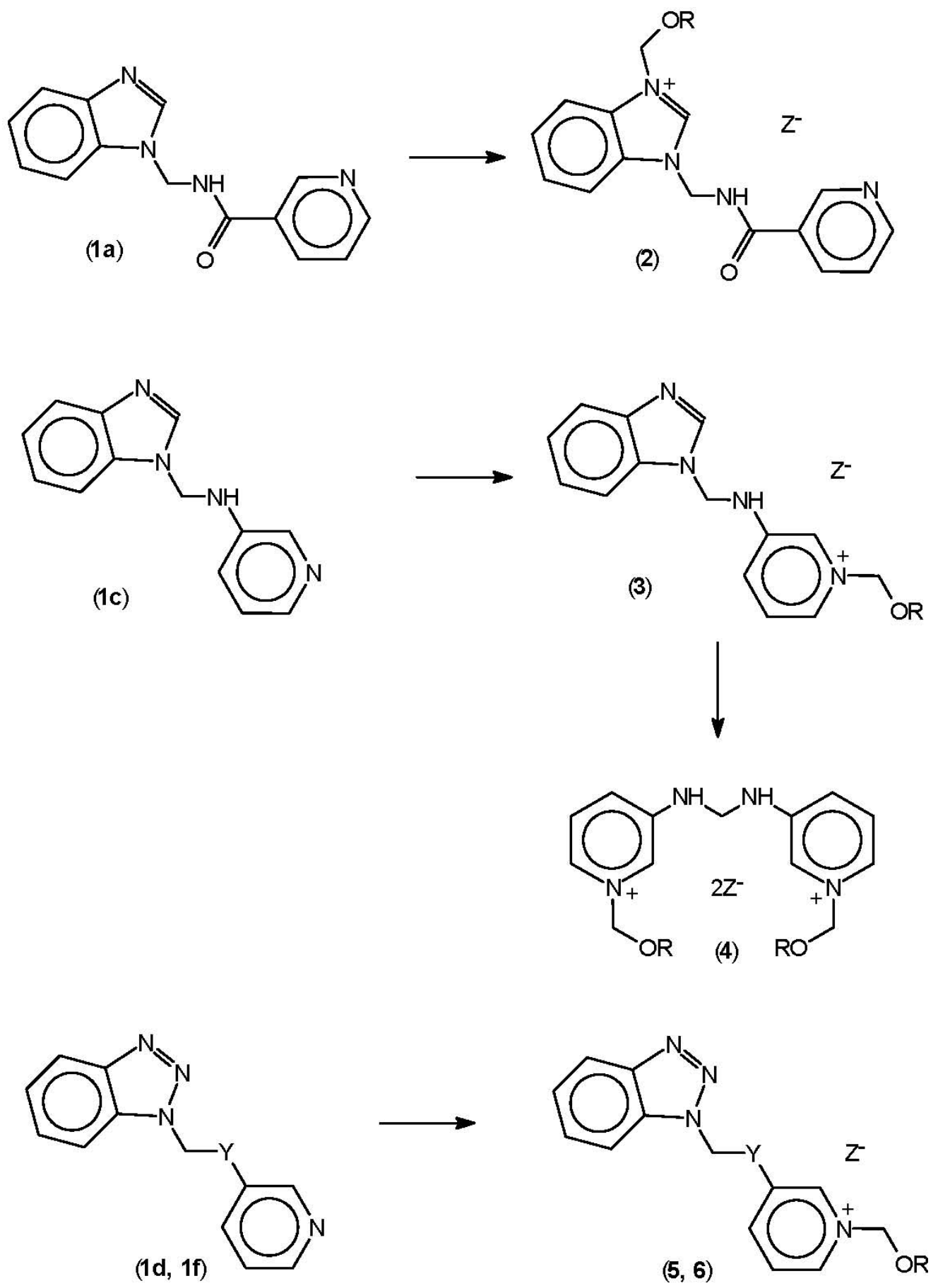

Scheme 2 
The structure of the quaternazation product depends on the $\mathrm{Y}$ group in compounds 1a and 1c. The strong electron-withdrawing group $(\mathrm{CONH})$ in the 3-position of the pyridine ring deactivates the nitrogen atom in pyridine. Chloromethylalkyl ether quaternarizes the N-3 of 1-substituted benzimidazole giving benzimidazolium chlorides 2 (Table 2). This study has shown that N-3 is nucleophilic enough to react with electrophiles.

Table 2. 1-Alkoxymethyl-3-[N-methyl-(3-pyridinecarbonyl)] benzimidazolium Salts 2

\begin{tabular}{lllll}
\hline Salt & $\mathrm{R}$ & $\mathrm{Z}$ & Yield (\%) & m.p. ${ }^{\mathrm{a}}\left({ }^{\circ} \mathrm{C}\right)$ \\
\hline $\mathbf{2 a}$ & $\mathrm{C}_{4} \mathrm{H}_{9}$ & $\mathrm{Cl}$ & 85 & $133-137^{\mathrm{b}}$ \\
$\mathbf{2 b}$ & $\mathrm{C}_{12} \mathrm{H}_{25}$ & $\mathrm{Cl}$ & 70 & $119-123^{\mathrm{b}}$ \\
$\mathbf{2 c}$ & $\mathrm{CycloC}_{12} \mathrm{H}_{25}$ & $\mathrm{Cl}$ & 70 & $163-165^{\mathrm{b}}$ \\
$\mathbf{2 d}$ & $\mathrm{C}_{3} \mathrm{H}_{7}$ & $\mathrm{BF}_{4}$ & 78 & $128-130$ \\
$\mathbf{2 e}$ & $\mathrm{C}_{8} \mathrm{H}_{17}$ & $\mathrm{BF}_{4}$ & 75 & $125-128$ \\
$\mathbf{2 f}$ & $\mathrm{C}_{12} \mathrm{H}_{25}$ & $\mathrm{I}$ & 80 & $111-112$ \\
$\mathbf{2 g}$ & $\mathrm{C}_{12} \mathrm{H}_{25}$ & $\mathrm{BF}_{4}$ & 83 & $127-128$ \\
$\mathbf{2 h}$ & $\mathrm{C}_{12} \mathrm{H}_{25}$ & $\mathrm{ClO}_{4}$ & 81 & $133-134$ \\
$\mathbf{2 i}$ & $\mathrm{CycloC}_{12} \mathrm{H}_{25}$ & $\mathrm{ClO}_{4}$ & 80 & $175-176$ \\
$\mathbf{2 j}$ & $\mathrm{CycloC}_{12} \mathrm{H}_{25}$ & $\mathrm{Br}$ & 80 & $164-166$ \\
$\mathbf{2 k}$ & $\mathrm{CycloC}_{12} \mathrm{H}_{25}$ & $\mathrm{I}$ & 78 & $154-156$ \\
$\mathbf{2 l}$ & $\mathrm{CycloC}_{12} \mathrm{H}_{25}$ & $\mathrm{NO}_{3}$ & 70 & $138-143$ \\
$\mathbf{2 m}$ & $\mathrm{CycloC}_{12} \mathrm{H}_{25}$ & $\mathrm{BF}_{4}$ & 70 & $138-140$ \\
\hline
\end{tabular}

a Solvent for recrystallization: $\mathrm{CHCl}_{3}: \mathrm{CH}_{3} \mathrm{CO}_{2} \mathrm{C}_{2} \mathrm{H}_{5}$ - 2a-2c; $\mathrm{CH}_{3} \mathrm{CO}_{2} \mathrm{C}_{2} \mathrm{H}_{5}$ - 2d-2m; bHygroscopic

The pyridinium salts 3-6 were prepared by the reaction of N-Mannich bases 1c, $1 \mathrm{~d}$ and $1 \mathrm{f}$ with chloromethylalkyl ethers. The reactions and the results are shown in Scheme 2 and Tables 3 and 4. 
Table 3. 1-Alkoxymethyl-3-(1-benzimidazolmethylamino)- pyridinium Salts 3 And Bis(1Alkoxymethyl- pyridinium) Salts 4

\begin{tabular}{|c|c|c|c|c|}
\hline Salt & $\mathrm{R}$ & Z & Yield (\%) & m.p..$^{\mathrm{a}}\left({ }^{\circ} \mathrm{C}\right)$ \\
\hline 3a & $\mathrm{C}_{3} \mathrm{H}_{7}$ & $\mathrm{Cl}$ & 82 & $143-145$ \\
\hline $\mathbf{3 b}$ & $\mathrm{C}_{8} \mathrm{H}_{17}$ & $\mathrm{Cl}$ & 70 & $141-143$ \\
\hline $3 c$ & $\mathrm{C}_{11} \mathrm{H}_{23}$ & $\mathrm{Cl}$ & 70 & $146-147$ \\
\hline 3d & $\mathrm{C}_{3} \mathrm{H}_{7}$ & $\mathrm{Br}$ & 70 & $143-144$ \\
\hline $3 e$ & $\mathrm{C}_{8} \mathrm{H}_{17}$ & $\mathrm{NO}_{3}$ & 70 & $118-120$ \\
\hline $3 f$ & $\mathrm{C}_{8} \mathrm{H}_{17}$ & I & 70 & $121-122$ \\
\hline $3 g$ & $\mathrm{C}_{8} \mathrm{H}_{17}$ & $\mathrm{BF}_{4}$ & 70 & $115-117$ \\
\hline $3 \mathbf{h}$ & $\mathrm{C}_{8} \mathrm{H}_{17}$ & $\mathrm{PF}_{6}$ & 75 & $109-112$ \\
\hline $3 \mathbf{i}$ & $\mathrm{C}_{8} \mathrm{H}_{17}$ & $\mathrm{ClO}_{4}$ & 73 & $108-110$ \\
\hline $3 \mathbf{j}$ & $\mathrm{C}_{12} \mathrm{H}_{25}$ & I & 70 & $107-109$ \\
\hline $3 k$ & $\mathrm{C}_{12} \mathrm{H}_{25}$ & $\mathrm{PF}_{6}$ & 70 & $111-114$ \\
\hline 31 & $\mathrm{C}_{12} \mathrm{H}_{25}$ & $\mathrm{ClO}_{4}$ & 80 & $114-116$ \\
\hline $4 a$ & $\mathrm{C}_{9} \mathrm{H}_{19}$ & $\mathrm{Cl}$ & 85 & $68-71$ \\
\hline $4 b$ & $\mathrm{C}_{11} \mathrm{H}_{23}$ & $\mathrm{Cl}$ & 84 & $72-73$ \\
\hline $4 c$ & $\mathrm{C}_{8} \mathrm{H}_{17}$ & $\mathrm{ClO}_{4}$ & 87 & $125-127$ \\
\hline $4 d$ & $\mathrm{C}_{8} \mathrm{H}_{17}$ & $\mathrm{BrO}_{3}$ & 85 & $129-130$ \\
\hline $4 e$ & $\mathrm{C}_{8} \mathrm{H}_{17}$ & I & 85 & $130-132$ \\
\hline $4 f$ & $\mathrm{C}_{8} \mathrm{H}_{17}$ & $\mathrm{Br}$ & 80 & $126-128$ \\
\hline $4 g$ & $\mathrm{C}_{12} \mathrm{H}_{25}$ & $\mathrm{PF}_{6}$ & 76 & $129-132$ \\
\hline $4 h$ & $\mathrm{C}_{12} \mathrm{H}_{25}$ & $\mathrm{SbF}_{6}$ & 73 & $92-94$ \\
\hline $4 i$ & $\mathrm{C}_{12} \mathrm{H}_{25}$ & I & 70 & $144-146$ \\
\hline
\end{tabular}

${ }^{a}$ Solvent for recrystallization: $\mathrm{MeOH} / \mathrm{Me} 2 \mathrm{CO}(1: 10)$ for $3 \mathrm{a}, 3 \mathrm{~b}, \mathrm{H} 2 \mathrm{O}$ for $3 \mathrm{c}, \mathrm{MeOH} / \mathrm{H} 2 \mathrm{O}(1: 1)$ for 3c, 3d, H2O-4a, 4b, MeOH 4c-4i

Symmetrically substituted bis(1-alkoxymethyl)pyridinium chlorides 4 were synthesised by trea-ting 1-alkoxymethyl-3-(1-benzimidazolmethylamino)pyridinium chloride $\mathbf{3}$ with chloromethyl-alkyl ether (Scheme 2). The formation of bispyridinium chloride is probably the result of the attack of chloromethylalkyl ether on the N-3 of benzimidazole ring to give an intermediate shown is Figure 2 which is unstable and quickly converted to bispyridinium chloride to replace the benzimidazole moiety. 


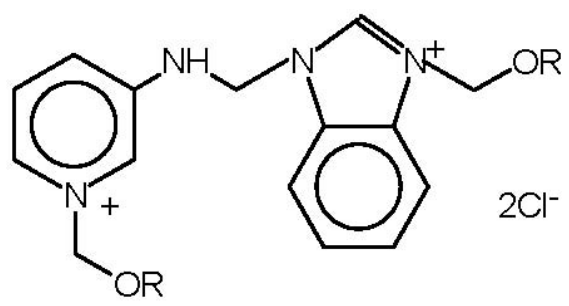

Figure 2

The substrate 3 was converted into the same derivative 4 by heating with hydrochloric acid.

Benzimidazole is a good leaving group in $\mathrm{N}-(1 H$-benzimidazolmethyl)-3pyridinecarboxamide 1a in basic and acidic solutions (Scheme 3). Hydrolysis is generally effected by heating with dilute hydrochloric acid. Treatment of compound 1a with alkoxides at $\mathrm{rt}$, displaces the benzimidazole anion giving a symmetrical diamide.

All of the salts 2-6 were prepared via metathesis of pyridinium or benzimidazolium chlorides with the appropriate inorganic salt in water solution (Tables 2, 3 and 4). In general, the chlorides were very hygroscopic.

Table 4. Pyridinium Salts 5,6

\begin{tabular}{cccccc}
\hline Salt & $\mathrm{R}$ & $\mathrm{Y}$ & $\mathrm{Z}$ & Yield(\%) & m.p. ${ }^{\mathrm{a}}\left({ }^{\circ} \mathrm{C}\right)$ \\
\hline $\mathbf{5 a}$ & $\mathrm{C}_{6} \mathrm{H}_{13}$ & $\mathrm{CONH}$ & $\mathrm{I}$ & 80 & $138-140$ \\
$\mathbf{5 b}$ & $\mathrm{C}_{8} \mathrm{H}_{17}$ & $\mathrm{CONH}$ & $\mathrm{Br}$ & 80 & $159-161$ \\
$\mathbf{6 a}$ & $\mathrm{C}_{3} \mathrm{H}_{7}$ & $\mathrm{NH}$ & $\mathrm{Cl}$ & 80 & $132-134$ \\
$\mathbf{6 b}$ & $\mathrm{C}_{4} \mathrm{H}_{9}$ & $\mathrm{NH}$ & $\mathrm{Cl}$ & 80 & $139-140$ \\
$\mathbf{6 c}$ & $\mathrm{C}_{5} \mathrm{H}_{11}$ & $\mathrm{NH}$ & $\mathrm{Cl}$ & 82 & $139-141$ \\
$\mathbf{6 d}$ & $\mathrm{C}_{6} \mathrm{H}_{13}$ & $\mathrm{NH}$ & $\mathrm{Cl}$ & 81 & $146-148$ \\
$\mathbf{6 e}$ & $\mathrm{C}_{7} \mathrm{H}_{15}$ & $\mathrm{NH}$ & $\mathrm{Cl}$ & 78 & $133-135$ \\
$\mathbf{6 f}$ & $\mathrm{C}_{8} \mathrm{H}_{17}$ & $\mathrm{NH}$ & $\mathrm{Cl}$ & 80 & $132-134$ \\
$\mathbf{6 g}$ & $\mathrm{C}_{9} \mathrm{H}_{19}$ & $\mathrm{NH}$ & $\mathrm{Cl}$ & 80 & $134-136$ \\
$\mathbf{6 h}$ & $\mathrm{C}_{10} \mathrm{H}_{21}$ & $\mathrm{NH}$ & $\mathrm{Cl}$ & 78 & $138-140$ \\
$\mathbf{6 i}$ & $\mathrm{C}_{11} \mathrm{H}_{23}$ & $\mathrm{NH}$ & $\mathrm{Cl}$ & 77 & $135-136$ \\
$\mathbf{6 j}$ & $\mathrm{C}_{12} \mathrm{H}_{25}$ & $\mathrm{NH}$ & $\mathrm{Cl}_{2}$ & 78 & $134-136$ \\
$\mathbf{6 k}$ & $\mathrm{C}_{3} \mathrm{H}_{7}$ & $\mathrm{NH}$ & $\mathrm{I}$ & 70 & $140-141$ \\
$\mathbf{6 l}$ & $\mathrm{C}_{3} \mathrm{H}_{7}$ & $\mathrm{NH}$ & $\mathrm{SbF}_{6}$ & 70 & $136-138$ \\
$\mathbf{6 m}$ & $\mathrm{C}_{4} \mathrm{H}_{9}$ & $\mathrm{NH}$ & $\mathrm{ClO}_{4}$ & 73 & $138-140$ \\
$\mathbf{6 n}$ & $\mathrm{C}_{4} \mathrm{H}_{9}$ & $\mathrm{NH}$ & $\mathrm{NO}_{3}$ & 70 & $134-136$ \\
$\mathbf{6 o}$ & $\mathrm{C}_{4} \mathrm{H}_{9}$ & $\mathrm{NH}$ & $\mathrm{PF}_{6}$ & 70 & $141-143$ \\
$\mathbf{6 p}$ & $\mathrm{C}_{4} \mathrm{H}_{9}$ & $\mathrm{NH}$ & $\mathrm{BF}_{4}$ & 72 & $144-145$ \\
$\mathbf{6 q}$ & $\mathrm{C}_{4} \mathrm{H}_{9}$ & $\mathrm{NH}$ & $\mathrm{I}$ & 73 & $145-147$ \\
\hline
\end{tabular}

${ }^{a}$ Solvent for recrystallization: $\mathrm{H}_{2} \mathrm{O}(\mathbf{5 a}, \mathbf{5 b}$ and $\mathbf{6 a - j}), \mathrm{MeOH}-\mathrm{H}_{2} \mathrm{O}-(\mathbf{6 k - 6 q})$. 
The conversions of the chlorides to the $\mathrm{Br}, \mathrm{I}, \mathrm{NO} 3, \mathrm{BrO} 3, \mathrm{ClO} 4, \mathrm{BF} 4, \mathrm{PF} 6$ and SbF6 salts led to some which were hydrophobic. The larger -size anions changed the character of the salt.

In conclusion, a procedure has been developed for the preparation of 3-substituted pyridinium and 1-substituted benzimidazolium salts. The work-up procedures are very simple, the products are easily purified and the yields are high.
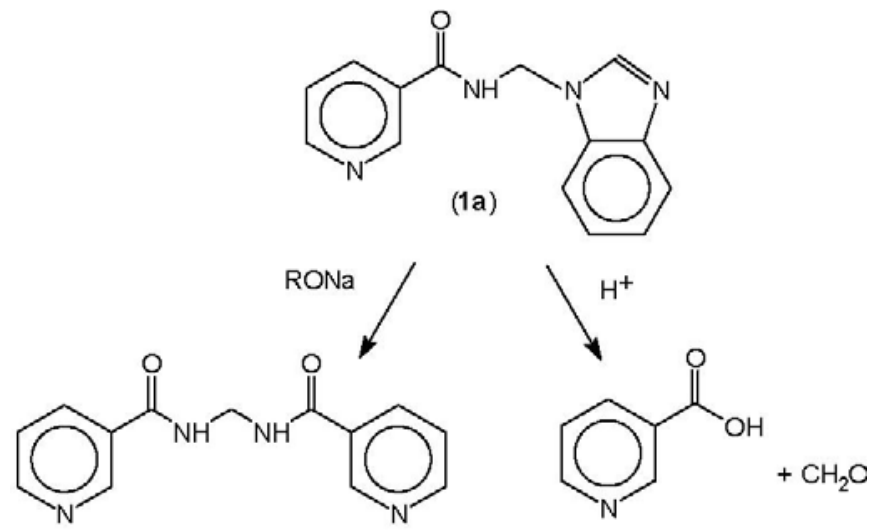

Scheme 3

\section{Experimental Section}

General Procedures. Melting points were determined on a Kofter hot stage apparatus and are uncorrected. The $1 \mathrm{H}$ NMR spectra were recorded with a Varian Model XL 300 Spectrometer at $300 \mathrm{MHz}$ with TMS as the standard. The 13C NMR spectra were recorded on the same instrument at $75 \mathrm{MHz}$. Elemental analyses was performed at the A. Mickiewicz University, Poznań. For all compounds 1-6 satisfactory microanalyses were obtained $\mathrm{C} \pm 0.31, \mathrm{H} \pm 0.30$ and $\mathrm{N} \pm 0.28$. Chloromethylalkyl ethers were prepared via the procedures, which were reported earlier $^{17}$.

The salts obtained were characterised by their $1 \mathrm{H}$ and 13C NMR spectra and by elemental analyses. The 1H and 13C NMR chemical shifts are summarised in Tables 5-10. The chemical shifts of the protons are anion-dependent in equivalent concentrations. This effect is strong for protons in the $\mathrm{Y}$ group $(\mathrm{H}-\mathrm{N})$ and weaker for the benzimidazole or benzotriazole ring protons. The H-N chemical shift decreases in the following order $\mathrm{Cl}>\mathrm{NO} 3>\mathrm{I}, \mathrm{ClO} 4, \mathrm{PF} 6>\mathrm{BF} 4$. This phenomenon has been noticed previously in the imidazole ring protons in 1,3 dialkylimidazolium salts $^{18}$.

\section{General Procedure for the condensation of nicotinamide}

Nicotinamide (12.2 g, $0.1 \mathrm{~mol})$, formaldehyde ( $3 \mathrm{~g}, 0.1 \mathrm{~mol}$, paraformaldehyde - powder) and benzimidazole or benzotriazole $(0.1 \mathrm{~mol})$ were refluxed in toluene $(200 \mathrm{~mL})$ to which two drops of concd. sulphuric acid had been added. The water formed during the reaction was removed azeotropically by a Dean-Stark apparatus. The toluene was then removed under reduced pressure 
(60 oC/30 Torr) and the resulting solids were recrystallized.

3-(Benzimidazolmethylamino)pyridine (1c): A mixture of 3-aminopyridine (9.4 g, $0.1 \mathrm{~mol})$, formaldehyde ( $3 \mathrm{~g}, 0.1 \mathrm{~mol}$, paraformaldehyde - powder) and benzimidazole (11.8 g, $0.1 \mathrm{~mol})$ was refluxed in EtOH $(100 \mathrm{~mL})$. After cooling to r.t. $\mathrm{H} 2 \mathrm{O}(50 \mathrm{~mL})$ was added, the product was collected by filtration and recrystallized.

\section{Pyridinium salts 3, 5 and 6. General procedure}

The 3-(Benzimidazolmethylamino)pyridine (1c) or 3-(benzotriazol-1-yl-methylamino)pyridine (1f) or N-(1 H-benzotriazol-1-ylmethyl)-3-pyridinecarboxamide (1d) (0.01 mol) was dissolved in anhydrous acetone and the corresponding chloromethylalkyl ether $(0.01 \mathrm{~mol})$ was added. The mixture was stirred at $\mathrm{rt}$ for 5-10 min. The precipitated solid was collected by filtration and recrystallised. A solution of the 1-alkoxymethylpyridinium chloride $(0.005 \mathrm{~mol})$ in $40 \mathrm{~mL}$ of $\mathrm{H} 2 \mathrm{O}$ and the appropriate inorganic salt $(0.02 \mathrm{~mol}, \mathrm{MZ})$ in $20 \mathrm{~mL}$ of $\mathrm{H} 2 \mathrm{O}$ were mixed at $\mathrm{rt}$ by stirring. The solid was collected by filtration and recrystallized.

\section{Benzimidazolium salts 2; General procedure}

The corresponding chloromethylalkyl ether $(0.01 \mathrm{~mol})$ was added to $\mathrm{N}-(1 \quad H$ benzimidazolmethyl)-3-pyridinecarboxamide (2.52 g, $0.01 \mathrm{~mol}, 1 \mathrm{a})$ in dry DMF (30 mL) by stirring for $1 \mathrm{~h}$ at r.t. The solvent was removed under reduced pressure. The crude product was washed with hexane and acetone and recrystallized. A sample of the benzimidazolium chloride $(0.01 \mathrm{~mol})$ in $40 \mathrm{~mL}$ of $\mathrm{H} 2 \mathrm{O}$ and the appropriate inorganic salt $(0.01 \mathrm{~mol}, \mathrm{MZ})$ in $20 \mathrm{~mL}$ was mixed at r.t. The solution was extracted by $50 \mathrm{~mL}$ of ethyl acetate, the extract was dried over $\mathrm{Na} 2 \mathrm{SO} 4$ and concentrated in vacuum. The resultant residue was recrystallized.

\section{Bispyridinium Salts 4; General procedure}

To 1-alkoxymethylpyridinium chloride $(0.01 \mathrm{~mol}, 3)$ in dry $\mathrm{CHCl} 3(40 \mathrm{~mL})$, chloromethylalkyl ether $(0.01 \mathrm{~mol})$ was added with stirring for $10 \mathrm{~min}$ at r.t. The solvent was removed under reduced pressure. The crude product was washed with hexane and recrystallized. A solution of prepared bispyridinuim chloride $(0.01 \mathrm{~mol})$ in $80 \mathrm{~mL}$ of $\mathrm{H}_{2} \mathrm{O}$ and a solution of inorganic salt $(0.02 \mathrm{~mol}, \mathrm{MZ})$ in $20 \mathrm{~mL}$ was mixed with stirring. The resulting product was collected by filtration and recrystallized. 
Table 5. ${ }^{1} \mathrm{H}$ NMR Spectral Data $(\delta, \mathrm{J} \text { in } \mathrm{Hz})^{\mathrm{a}}$ Benzimidazolium Salts 2

\begin{tabular}{|c|c|c|c|c|c|c|}
\hline Salt & $\mathrm{NH}$ & Pyridine & $\mathrm{NCH}_{2} \mathrm{~N}$ & Benzimidazole & $\mathrm{NCH}_{2} \mathrm{O}$ & $\mathrm{R}$ \\
\hline $2 b$ & $\begin{array}{l}11.25 \\
(\mathrm{t}, \\
J=6.0)\end{array}$ & 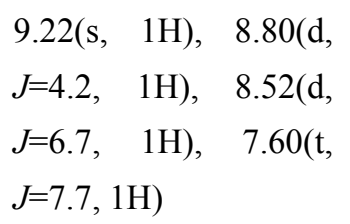 & $\begin{array}{l}6.08 \\
(\mathrm{~d}, \\
J=7.4)\end{array}$ & $\begin{array}{l}10.38(\mathrm{~s}, \quad 1 \mathrm{H}), \quad 8.58(\mathrm{~d}, \\
J=6.2,1 \mathrm{H}), 8.08(\mathrm{~d}, J=5.8 \\
1 \mathrm{H}), 7.74(\mathrm{~m}, 2 \mathrm{H})\end{array}$ & $6.06(\mathrm{~s})$ & $\begin{array}{l}3.57(\mathrm{t}, J=6.3,2 \mathrm{H}), \\
1.45(\mathrm{~m}, 2 \mathrm{H}), 1.13(\mathrm{~m}, \\
18 \mathrm{H}), 0.85(\mathrm{t}, J=6.6, \\
3 \mathrm{H})\end{array}$ \\
\hline 2d & $\begin{array}{l}10.10(\mathrm{t}, \\
J=6.0)\end{array}$ & $\begin{array}{l}9.07(\mathrm{~s}, \quad 1 \mathrm{H}), \quad 8.78(\mathrm{~d}, \\
J=4.8, \quad 1 \mathrm{H}), \quad 8.23(\mathrm{~m}, \\
1 \mathrm{H}), \quad 7.58(\mathrm{t}, \quad J=8.0, \\
1 \mathrm{H})\end{array}$ & $\begin{array}{l}6.08 \quad(\mathrm{~d}, \\
J=6.0)\end{array}$ & $\begin{array}{l}10.10(\mathrm{~s}, 1 \mathrm{H}), 8.23(\mathrm{~m}, 1 \mathrm{H}), \\
8.09(\mathrm{~d}, \quad J=7.0, \quad 1 \mathrm{H}), \\
7.77(\mathrm{~m}, 2 \mathrm{H})\end{array}$ & $6.01(\mathrm{~s})$ & $\begin{array}{l}3.55(\mathrm{t}, J=6.3,2 \mathrm{H}), \\
1.53(\mathrm{~m}, 2 \mathrm{H}), 0.81(\mathrm{t}, \\
J=6.7,3 \mathrm{H})\end{array}$ \\
\hline $2 \mathrm{e}$ & $\begin{array}{l}10.08(\mathrm{t}, \\
J=6.0)\end{array}$ & $\begin{array}{l}9.05(\mathrm{~s}, \quad 1 \mathrm{H}), \quad 8.77(\mathrm{~d}, \\
J=4.8, \quad 1 \mathrm{H}), \quad 8.23(\mathrm{~m}, \\
1 \mathrm{H}), \quad 7.56(\mathrm{t}, \quad J=7.8, \\
1 \mathrm{H})\end{array}$ & $\begin{array}{l}6.05 \quad(\mathrm{~d}, \\
J=6.3)\end{array}$ & $\begin{array}{l}10.08(\mathrm{~s}, 1 \mathrm{H}), 8.23(\mathrm{~m}, 1 \mathrm{H}), \\
8.10(\mathrm{~d}, \quad J=7.4, \quad 1 \mathrm{H}), \\
7.75(\mathrm{~m}, 2 \mathrm{H})\end{array}$ & $6.00(\mathrm{~s})$ & $\begin{array}{l}3.56(\mathrm{t}, \quad J=6.3,2 \mathrm{H}), \\
1.47(\mathrm{~m}, 2 \mathrm{H}), 1.14(\mathrm{~m}, \\
10 \mathrm{H}), 0.81(\mathrm{t}, J=6.7, \\
3 \mathrm{H})\end{array}$ \\
\hline $2 f$ & $\begin{array}{l}10.16(\mathrm{t}, \\
J=6.0)\end{array}$ & $\begin{array}{l}9.07(\mathrm{~s}, \quad 1 \mathrm{H}), \quad 8.78(\mathrm{~d}, \\
J=5.0, \quad 1 \mathrm{H}), \quad 8.25(\mathrm{~m}, \\
1 \mathrm{H}), \quad 7.58(\mathrm{t}, \quad J=7.8, \\
1 \mathrm{H})\end{array}$ & $\begin{array}{l}6.08 \quad(\mathrm{~d}, \\
J=6.0)\end{array}$ & $\begin{array}{l}10.16(\mathrm{~s}, 1 \mathrm{H}), 8.25(\mathrm{~m}, 1 \mathrm{H}), \\
8.08(\mathrm{~d}, \quad J=6.0, \quad 1 \mathrm{H}), \\
7.77(\mathrm{~m}, 2 \mathrm{H})\end{array}$ & $6.01(\mathrm{~s})$ & $\begin{array}{l}3.56(\mathrm{t}, J=6.3,2 \mathrm{H}), \\
1.47(\mathrm{~m}, 2 \mathrm{H}), 1.14(\mathrm{~m}, \\
18 \mathrm{H}), 0.86(\mathrm{t}, J=6.7, \\
3 \mathrm{H})\end{array}$ \\
\hline $2 \mathrm{~g}$ & $\begin{array}{l}10.21(\mathrm{t}, \\
J=6.0)\end{array}$ & $\begin{array}{l}9.07(\mathrm{~s}, \quad 1 \mathrm{H}), \quad 8.78(\mathrm{~d}, \\
J=3.6, \quad 1 \mathrm{H}), \quad 8.25(\mathrm{~m}, \\
1 \mathrm{H}), \quad 7.58(\mathrm{t}, \quad J=7.8, \\
1 \mathrm{H})\end{array}$ & $\begin{array}{l}6.08 \quad(\mathrm{~d}, \\
J=6.0)\end{array}$ & $\begin{array}{l}10.16(\mathrm{~s}, 1 \mathrm{H}), 8.25(\mathrm{~m}, 1 \mathrm{H}), \\
8.08(\mathrm{~d}, \quad J=7.1, \quad 1 \mathrm{H}), \\
7.77(\mathrm{~m}, 2 \mathrm{H})\end{array}$ & $6.01(\mathrm{~s})$ & $\begin{array}{l}3.56(\mathrm{t}, J=6.3,2 \mathrm{H}), \\
1.47(\mathrm{~m}, 2 \mathrm{H}), 1.14(\mathrm{~m}, \\
18 \mathrm{H}), 0.86(\mathrm{t}, J=6.7, \\
3 \mathrm{H})\end{array}$ \\
\hline $2 \mathrm{~h}$ & $\begin{array}{l}10.21(\mathrm{t}, \\
J=6.0)\end{array}$ & $\begin{array}{l}9.07(\mathrm{~s}, \quad 1 \mathrm{H}), \quad 8.78(\mathrm{~d}, \\
J=3.6, \quad 1 \mathrm{H}), \quad 8.25(\mathrm{~m}, \\
1 \mathrm{H}), \quad 7.58(\mathrm{t}, \quad J=7.7, \\
1 \mathrm{H})\end{array}$ & $\begin{array}{l}6.08 \quad(\mathrm{~d}, \\
J=6.0)\end{array}$ & $\begin{array}{l}10.16(\mathrm{~s}, 1 \mathrm{H}), 8.25(\mathrm{~m}, 1 \mathrm{H}), \\
8.08(\mathrm{~d}, \quad J=7.1, \quad 1 \mathrm{H}), \\
7.77(\mathrm{~m}, 2 \mathrm{H})\end{array}$ & $6.01(\mathrm{~s})$ & $\begin{array}{l}3.56(\mathrm{t}, J=6.3,2 \mathrm{H}), \\
1.47(\mathrm{~m}, 2 \mathrm{H}), 1.14(\mathrm{~m}, \\
18 \mathrm{H}), 0.86(\mathrm{t}, J=6.7, \\
3 \mathrm{H})\end{array}$ \\
\hline $2 m$ & $\begin{array}{l}10.23(\mathrm{t}, \\
J=6.0)\end{array}$ & $\begin{array}{l}9.06(\mathrm{~s}, \quad 1 \mathrm{H}), \quad 8.78(\mathrm{~d}, \\
J=4.8, \quad 1 \mathrm{H}), \quad 8.25(\mathrm{~m}, \\
1 \mathrm{H}), \quad 7.58(\mathrm{t}, \quad J=8.0, \\
1 \mathrm{H})\end{array}$ & $\begin{array}{l}6.08 \quad(\mathrm{~d}, \\
J=6.0)\end{array}$ & $\begin{array}{l}10.21(\mathrm{~s}, 1 \mathrm{H}), 8.25(\mathrm{~m}, 1 \mathrm{H}), \\
8.07(\mathrm{~d}, \quad J=7.8, \quad 1 \mathrm{H}), \\
7.78(\mathrm{~m}, 2 \mathrm{H})\end{array}$ & $6.03(\mathrm{~s})$ & $\begin{array}{l}3.63(\mathrm{~m}, 1 \mathrm{H}), 1.54(\mathrm{~m}, \\
2 \mathrm{H}), 1.40(\mathrm{~m}, 2 \mathrm{H}), \\
1.14(\mathrm{~m}, 18 \mathrm{H})\end{array}$ \\
\hline
\end{tabular}

${ }^{\mathrm{a}}$ Solvent DMSO- $d_{6}$ 
Table 6. ${ }^{13}$ C NMR Spectral Data Benzimidazolium Salts $\mathbf{2}^{\mathrm{a}}$

\begin{tabular}{|c|c|c|c|c|c|c|}
\hline Salt & $\mathrm{C}=\mathrm{O}$ & Pyridine & $\mathrm{NCH}_{2} \mathrm{~N}$ & Benzimidazole & $\mathrm{NCH}_{2} \mathrm{O}$ & $\mathrm{R}$ \\
\hline $2 b$ & 165.8 & $\begin{array}{l}152.2, \quad 148.3, \\
136.2,128.2,123.9\end{array}$ & 51.81 & $\begin{array}{l}143.9, \quad 130.6, \quad 130.5 \\
127.0,114.6,114.2\end{array}$ & 76.76 & $\begin{array}{l}69.0,31.3,29.0, \quad 28.98, \\
28.92,28.89,28.7,28.5, \\
28.0,25.2,22.1,14.0\end{array}$ \\
\hline 2d & 166.2 & $\begin{array}{l}152.7,148.4,135.2, \\
128.1,123.5\end{array}$ & 51.76 & $\begin{array}{lll}143.3, & 130.5, & 130.4, \\
127.0, & 126.9, & 114.0, \\
113.9 & & \end{array}$ & 76.78 & $70.79,21.83,9.99$ \\
\hline $2 e$ & 166.2 & $\begin{array}{l}152.7, \quad 148.4, \\
135.1,128.1,123.5\end{array}$ & 51.76 & $\begin{array}{l}143.3, \quad 130.5, \quad 130.4 \\
126.9,114.1,113.9\end{array}$ & 76.76 & $\begin{array}{l}69.1,30.9,28.4,28.3,28.2 \\
25.1,21.8,13.7\end{array}$ \\
\hline $2 f$ & 166.2 & $\begin{array}{l}152.9, \quad 148.6, \\
135.3,128.0,123.6\end{array}$ & 51.84 & $\begin{array}{l}143.6, \quad 130.5, \quad 130.4 \\
127.0,114.2,114.1\end{array}$ & 76.82 & 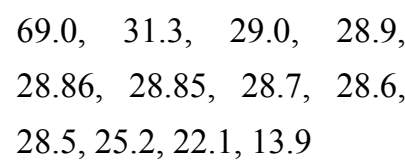 \\
\hline $2 g$ & 166.3 & $\begin{array}{l}152.8, \quad 148.6, \\
135.3,128.1,123.7\end{array}$ & 51.86 & $\begin{array}{l}\text { 143.6, 130.6, 130.5, } \\
127.1,114.2,114.1\end{array}$ & 76.85 & $\begin{array}{l}69.1,31.3,29.0,28.98, \\
28.93,28.91,28.7,28.6, \\
28.5,25.3,22.1,13.9\end{array}$ \\
\hline $2 \mathrm{~h}$ & 166.3 & $\begin{array}{l}152.9, \quad 148.6, \\
135.3,128.1,123.7\end{array}$ & 51.86 & $\begin{array}{l}\text { 143.6, 130.6, 130.5, } \\
127.1,114.2,114.1\end{array}$ & 76.84 & $\begin{array}{l}69.1,31.3,29.0,28.98 \\
28.92,28.89, .28 .7,28.6 \\
28.5,25.3,22.1,13.9\end{array}$ \\
\hline $2 m$ & 166.3 & $\begin{array}{l}152.9, \quad 148.6, \\
135.2,128.1,123.7\end{array}$ & 51.73 & $\begin{array}{l}143.2, \quad 130.6, \quad 130.4 \\
127.1,114.3,114.1\end{array}$ & 76.49 & $\begin{array}{l}75.2,28.5,23.6,23.4,22.6, \\
20.3\end{array}$ \\
\hline
\end{tabular}

${ }^{\mathrm{a}}$ Solvent DMSO- $d_{6}$

Table 7. ${ }^{1} \mathrm{H}$ NMR Spectral Data $(\delta, \mathrm{J} \text { in } \mathrm{Hz})^{\mathrm{a}}$ of Pyridinium Salts 3 and Bispyridinium Salts 4

\begin{tabular}{|c|c|c|c|c|c|c|}
\hline Salt & $\mathrm{NH}$ & Pyridine & $\mathrm{NCH}_{2} \mathrm{~N}$ & Benzimidazole & $\mathrm{NCH}_{2} \mathrm{O}$ & $\mathrm{R}$ \\
\hline $3 \mathbf{a}$ & - & 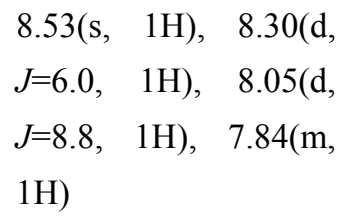 & $5.95(\mathrm{~s})$ & $\begin{array}{l}8.51(\mathrm{~s}, 1 \mathrm{H}), 7.65(\mathrm{~d}, J=7.7, \\
1 \mathrm{H}), 7.84(\mathrm{~m}, 1 \mathrm{H}), 7.32(\mathrm{~m}, \\
2 \mathrm{H})\end{array}$ & $5.81(\mathrm{~s})$ & $\begin{array}{l}3.46(\mathrm{t}, \quad J=6.6,2 \mathrm{H}), \\
1.55(\mathrm{~m}, 2 \mathrm{H}), 0.85(\mathrm{t}, \\
J=7.4,3 \mathrm{H})\end{array}$ \\
\hline $3 \mathbf{b}$ & $\begin{array}{l}8.98 \quad(\mathrm{t}, \\
J=6.9)\end{array}$ & 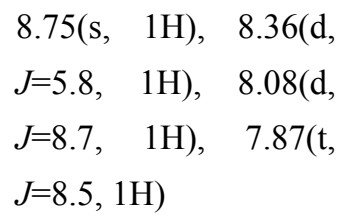 & $\begin{array}{l}5.91 \quad(\mathrm{~d}, \\
J=6.9)\end{array}$ & $\begin{array}{l}8.69(\mathrm{~s}, 1 \mathrm{H}), 7.94(\mathrm{~d}, J=7.4 \\
1 \mathrm{H}), 7.64(\mathrm{~d}, J=7.7,1 \mathrm{H}), \\
7.25(\mathrm{~m}, 2 \mathrm{H})\end{array}$ & $5.82(\mathrm{~s})$ & $\begin{array}{l}3.45(\mathrm{t}, \quad J=6.4,2 \mathrm{H}), \\
1.45(\mathrm{~m}, 2 \mathrm{H}), 1.22(\mathrm{~m}, \\
10 \mathrm{H}), 0.84(\mathrm{t}, J=6.8, \\
3 \mathrm{H})\end{array}$ \\
\hline $3 c$ & $\begin{array}{l}9.09 \quad(\mathrm{t}, \\
J=6.9)\end{array}$ & 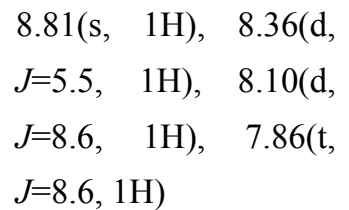 & $\begin{array}{l}5.91 \quad(\mathrm{~d}, \\
J=6.9)\end{array}$ & $\begin{array}{l}8.71(\mathrm{~s}, 1 \mathrm{H}), 7.96(\mathrm{~d}, J=7.4, \\
1 \mathrm{H}), 7.62(\mathrm{~d}, J=7.4,1 \mathrm{H}), \\
7.23(\mathrm{~m}, 2 \mathrm{H})\end{array}$ & $5.83(\mathrm{~s})$ & $\begin{array}{l}3.48(\mathrm{t}, \quad J=6.5,2 \mathrm{H}), \\
1.46(\mathrm{~m}, 2 \mathrm{H}), 1.21(\mathrm{~m}, \\
16 \mathrm{H}), 0.85(\mathrm{t}, J=6.7, \\
3 \mathrm{H})\end{array}$ \\
\hline
\end{tabular}


Table 7. Continued

\begin{tabular}{|c|c|c|c|c|c|c|c|c|c|}
\hline $3 f$ & $\begin{array}{l}8.52 \quad(\mathrm{t}, \\
J=6.7)\end{array}$ & $\begin{array}{l}8.60(\mathrm{~s}, \quad 1 \mathrm{H}), \\
J=5.8, \quad 1 \mathrm{H}), \\
J=8.7, \quad 1 \mathrm{H}), \\
J=8.5,1 \mathrm{H})\end{array}$ & $\begin{array}{l}8.41(\mathrm{~d}, \\
8.09(\mathrm{~d}, \\
7.93(\mathrm{t},\end{array}$ & $\begin{array}{l}5.94 \quad(\mathrm{~d}, \\
J=6.7)\end{array}$ & $\begin{array}{l}8.62(\mathrm{~s}, \\
J=7.9, \\
J=7.6, \\
2 \mathrm{H})\end{array}$ & $\begin{array}{l}1 \mathrm{H}), \\
1 \mathrm{H}), \\
1 \mathrm{H}),\end{array}$ & $\begin{array}{c}7.87(\mathrm{~d}, \\
7.67 \mathrm{~d}, \\
7.28(\mathrm{~m},\end{array}$ & $5.84(\mathrm{~s})$ & $\begin{array}{l}3.50(\mathrm{t}, \quad J=6.4, \quad 2 \mathrm{H}), \\
1.46(\mathrm{~m}, 2 \mathrm{H}), \quad 1.23(\mathrm{~m}, \\
10 \mathrm{H}), \quad 0.85(\mathrm{t}, \quad J=6.7, \\
3 \mathrm{H})\end{array}$ \\
\hline $3 g$ & $\begin{array}{l}8.49 \quad(\mathrm{t}, \\
J=6.6)\end{array}$ & $\begin{array}{l}8.56(\mathrm{~s}, 1 \mathrm{H}), 8.3( \\
J=5.8), \quad 8.05(\mathrm{c} \\
J=8.8), \quad 7.90(\mathrm{t}, \\
1 \mathrm{H})\end{array}$ & $\begin{array}{r}6(\mathrm{~d}, 1 \mathrm{H}, \\
\mathrm{d}, \quad 1 \mathrm{H}, \\
J=8.8,\end{array}$ & $\begin{array}{l}5.91 \quad(\mathrm{~d}, \\
J=6.9)\end{array}$ & $\begin{array}{l}8.52(\mathrm{~s}, \\
J=7.7, \\
J=7.4, \\
2 \mathrm{H})\end{array}$ & $\begin{array}{l}1 \mathrm{H}), \\
1 \mathrm{H}), \\
1 \mathrm{H}),\end{array}$ & $\begin{array}{c}7.84(\mathrm{~d}, \\
7.67(\mathrm{~d}, \\
7.27(\mathrm{~m},\end{array}$ & $5.79(\mathrm{~s})$ & $\begin{array}{l}3.49(\mathrm{t}, \quad J=6.3, \quad 2 \mathrm{H}), \\
1.48(\mathrm{~m}, 2 \mathrm{H}), \quad 1.22(\mathrm{~m}, \\
10 \mathrm{H}), \quad 0.85(\mathrm{t}, \quad J=6.4, \\
3 \mathrm{H})\end{array}$ \\
\hline $3 h$ & 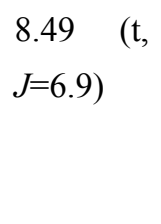 & $\begin{array}{l}8.55(\mathrm{~s}, \quad 1 \mathrm{H}), \\
J=5.8, \quad 1 \mathrm{H}), \\
J=8.7, \quad 1 \mathrm{H}), \\
J=8.7,1 \mathrm{H})\end{array}$ & $\begin{array}{l}8.35(\mathrm{~d}, \\
8.03(\mathrm{~d}, \\
7.89(\mathrm{t},\end{array}$ & $\begin{array}{l}5.90 \quad(\mathrm{~d}, \\
J=6.6)\end{array}$ & $\begin{array}{l}8.52(\mathrm{~s}, \\
J=8.0, \\
J=7.7, \\
2 \mathrm{H})\end{array}$ & $\begin{array}{l}1 \mathrm{H}), \\
1 \mathrm{H}), \\
1 \mathrm{H}),\end{array}$ & $\begin{array}{c}7.83(\mathrm{~d}, \\
7.66(\mathrm{~d}, \\
7.23(\mathrm{~m},\end{array}$ & $5.78(\mathrm{~s})$ & $\begin{array}{l}3.48(\mathrm{t}, \quad J=6.6, \quad 2 \mathrm{H}), \\
1.48(\mathrm{~m}, 2 \mathrm{H}), \quad 1.22(\mathrm{~m}, \\
10 \mathrm{H}), \quad 0.85(\mathrm{t}, \quad J=6.7, \\
3 \mathrm{H})\end{array}$ \\
\hline $4 b$ & $\begin{array}{l}9.51 \quad(\mathrm{t}, \\
J=6.0 \\
2 \mathrm{H})\end{array}$ & $\begin{array}{l}9.38(\mathrm{~s}, \quad 2 \mathrm{H}), \\
J=8.5, \quad 2 \mathrm{H}), \\
J=6.0, \quad 2 \mathrm{H}), \\
J=8.5,2 \mathrm{H})\end{array}$ & $\begin{array}{l}8.16(\mathrm{~d}, \\
7.97 \mathrm{~d}, \\
7.65(\mathrm{t},\end{array}$ & $\begin{array}{l}4.87 \quad(\mathrm{t}, \\
J=5.5 \\
2 \mathrm{H})\end{array}$ & - & & & $\begin{array}{l}6.07 \quad(\mathrm{~s}, \\
4 \mathrm{H})\end{array}$ & $\begin{array}{l}3.68(\mathrm{t}, \quad J=6.4, \quad 4 \mathrm{H}), \\
1.61(\mathrm{~m}, 4 \mathrm{H}), \quad 1.32(\mathrm{~m}, \\
32 \mathrm{H}), \quad 0.88(\mathrm{t}, \quad J=6.6, \\
6 \mathrm{H})\end{array}$ \\
\hline $4 c$ & $\begin{array}{l}8.01 \quad(\mathrm{t}, \\
J=5.5 \\
2 \mathrm{H})\end{array}$ & $\begin{array}{l}8.35(\mathrm{~m}, 4 \mathrm{H}), \\
4 \mathrm{H})\end{array}$ & 7.89(m, & $\begin{array}{l}4.84 \quad(\mathrm{t}, \\
J=5.4 \\
2 \mathrm{H})\end{array}$ & - & & & $\begin{array}{l}5.79(\mathrm{~s}, \\
4 \mathrm{H})\end{array}$ & $\begin{array}{l}3.56(\mathrm{t}, \quad J=6.6, \quad 4 \mathrm{H}), \\
1.52(\mathrm{~m}, 4 \mathrm{H}), \quad 1.25(\mathrm{~m}, \\
20 \mathrm{H}), \quad 0.85(\mathrm{t}, \quad J=6.7, \\
6 \mathrm{H})\end{array}$ \\
\hline $4 e$ & $\begin{array}{l}8.58 \quad(\mathrm{t}, \\
J=6.0 \\
2 \mathrm{H})\end{array}$ & $\begin{array}{l}9.17(\mathrm{~s}, \quad 2 \mathrm{H}), \\
J=8.6, \quad 2 \mathrm{H}), \\
J=5.8, \quad 2 \mathrm{H}), \\
J=8.8,2 \mathrm{H})\end{array}$ & $\begin{array}{l}8.33(\mathrm{~d}, \\
8.12(\mathrm{~d}, \\
7.69(\mathrm{t},\end{array}$ & $\begin{array}{l}4.87 \quad(\mathrm{t}, \\
J=5.9 \\
2 \mathrm{H})\end{array}$ & - & & & $\begin{array}{l}6.18(\mathrm{~s}, \\
4 \mathrm{H})\end{array}$ & $\begin{array}{l}3.74(\mathrm{t}, \quad J=6.5, \quad 4 \mathrm{H}), \\
1.64(\mathrm{~m}, 4 \mathrm{H}), \quad 1.31(\mathrm{~m}, \\
20 \mathrm{H}), \quad 0.87(\mathrm{t}, \quad J=6.7, \\
6 \mathrm{H})\end{array}$ \\
\hline $4 \mathbf{i}$ & $\begin{array}{l}8.02 \quad(\mathrm{t}, \\
J=5.5 \\
2 \mathrm{H})\end{array}$ & $\begin{array}{l}8.35(\mathrm{~m}, \quad 4 \mathrm{H}) \\
4 \mathrm{H})\end{array}$ & 7.89(m, & $\begin{array}{l}4.83 \quad(\mathrm{t}, \\
J=5.5 \\
2 \mathrm{H})\end{array}$ & - & & & $\begin{array}{l}5.78(\mathrm{~s}, \\
4 \mathrm{H})\end{array}$ & $\begin{array}{l}3.54(\mathrm{t}, \quad J=6.5, \quad 4 \mathrm{H}), \\
1.51(\mathrm{~m}, \quad 4 \mathrm{H}), \quad 1.24(\mathrm{~m}, \\
36 \mathrm{H}), \quad 0.86(\mathrm{t}, \quad J=6.7, \\
6 \mathrm{H})\end{array}$ \\
\hline
\end{tabular}

${ }^{\mathrm{a}}$ Solvent: $\mathrm{CD}_{3} \mathrm{OD}-\mathbf{3 a} ; \mathrm{CDCl}_{3}-\mathbf{4 b}, \mathbf{4 e}$; $\mathrm{DMSO}-d_{6}-\mathbf{3 b}, \mathbf{3 c}, \mathbf{3 f}, \mathbf{3 g}, \mathbf{3 h}, \mathbf{4 c}, \mathbf{4 i}$ 
Table 8. ${ }^{13} \mathrm{C}$ NMR Spectral Data ${ }^{\text {a }}$ of Pyridinium Salts 3 and Bispyridinium Salts 4

\begin{tabular}{|c|c|c|c|c|c|}
\hline Salt & Pyridine & $\mathrm{NCH}_{2} \mathrm{~N}$ & Benzimidazole & $\mathrm{NCH}_{2} \mathrm{O}$ & $\mathrm{R}$ \\
\hline $\mathbf{3 a}$ & $\begin{array}{l}147.8,133.0,130.1, \\
129.3,127.5\end{array}$ & 53.94 & $\begin{array}{l}144.5,144.3,134.2,124.9, \\
124.2,120.4,112.1\end{array}$ & 90.73 & $73.9,23.5,10.6$ \\
\hline $\mathbf{3 b}$ & $\begin{array}{l}146.0,131.8,128.5 \\
128.0,126.6\end{array}$ & 51.89 & $\begin{array}{l}144.0,143.5,133.0,122.6 \\
122.1,119.4,111.4\end{array}$ & 88.62 & $70.1,31.2,28.6,25.2,22.1,14.0$ \\
\hline $3 c$ & $\begin{array}{l}146.0,131.8,128.4, \\
128.0,126.6\end{array}$ & 51.88 & $\begin{array}{l}143.9, \quad 122.6,122.0,119.5, \\
111.3\end{array}$ & 88.62 & $\begin{array}{l}70.1,31.3,28.9,28.6,28.6, \\
25.1,22.1,13.9\end{array}$ \\
\hline $3 f$ & $\begin{array}{l}146.0,132.0,128.6 \\
128.2,126.5\end{array}$ & 52.00 & $\begin{array}{l}144.0,143.7,133.2,122.7 \\
122.1,119.6,111.2\end{array}$ & 88.68 & $\begin{array}{l}70.2,31.1,28.5,28.45,25.1, \\
22.0,13.8\end{array}$ \\
\hline $3 g$ & $\begin{array}{l}145.9,131.9,128.4, \\
128.1,126.5\end{array}$ & 51.96 & $122.7,122.1,119.6,111.1$ & 88.01 & $70.2,31.2,28.6,25.2,22.1,14.0$ \\
\hline $3 \mathbf{h}$ & $\begin{array}{l}145.9,131.9,128.4, \\
128.1,126.5\end{array}$ & 51.95 & $122.7,122.1,119.6,111.1$ & 88.78 & $70.2,31.2,28.6,25.2,22.1,14.0$ \\
\hline $4 b$ & $\begin{array}{l}146.7,130.7,127.5, \\
127.0,125.8\end{array}$ & 50.00 & - & 88.83 & $\begin{array}{l}71.7,31.7,29.4,29.3,29.1, \\
29.0,25.6,22.5,13.9\end{array}$ \\
\hline $4 c$ & $\begin{array}{l}146.1,130.8,128.3 \\
127.5,126.0\end{array}$ & 50.70 & - & 88.61 & $\begin{array}{l}70.1, \quad 31.1,28.6,28.5, \quad 25.2, \\
22.0,13.9\end{array}$ \\
\hline $4 e$ & $\begin{array}{l}145.8,130.4,128.4, \\
127.0,124.6\end{array}$ & 49.01 & - & 88.31 & $\begin{array}{l}71.6, \quad 31.4, \quad 29.0, \quad 28.9, \quad 28.8, \\
25.5,22.3,13.8\end{array}$ \\
\hline $4 \mathbf{i}$ & $\begin{array}{l}146.3,131.4,128.5 \\
127.8,126.3\end{array}$ & 50.66 & - & 88.74 & $\begin{array}{lllll}70.2, & 31.3, & 29.1, & 29.0, & 29.0, \\
28.8, & 28.7, & 28.65, & 25.3, & 22.1, \\
14.0 & & & & \end{array}$ \\
\hline
\end{tabular}

${ }^{\mathrm{a}}$ Solvent: $\mathrm{CD}_{3} \mathrm{OD}-\mathbf{3 a} ; \mathrm{CDCl}_{3}-\mathbf{4 b}, \mathbf{4 e}$; DMSO- $d_{6}-\mathbf{3 b}, \mathbf{3 c}, \mathbf{3 f}, \mathbf{3 g}, \mathbf{3 h}, \mathbf{4} \mathbf{c}, \mathbf{4} \mathbf{i}$

Table 9. ${ }^{1} \mathrm{H}$ NMR Spectral Data $(\delta, \mathrm{J} \text { in } \mathrm{Hz})^{\mathrm{a}}$ of Pyridinium Salts 5,6

\begin{tabular}{|c|c|c|c|c|c|c|}
\hline Salt & $\mathrm{NH}$ & Pyridine & $\mathrm{NCH}_{2} \mathrm{~N}$ & Benzotriazole & $\mathrm{NCH}_{2} \mathrm{O}$ & $\mathrm{R}$ \\
\hline $5 a$ & $\begin{array}{l}10.61 \\
(\mathrm{t}, J=6)\end{array}$ & $\begin{array}{l}9.53(\mathrm{~s}, \quad 1 \mathrm{H}), \quad 9.26(\mathrm{~d}, \\
J=6,1 \mathrm{H}), 9.07(\mathrm{~d}, J=7 \\
1 \mathrm{H}), 8.37(\mathrm{t}, J=7,1 \mathrm{H})\end{array}$ & $\begin{array}{l}6.34 \quad(\mathrm{~d}, \\
J=6)\end{array}$ & $\begin{array}{l}8.09(\mathrm{~d}, J=9,2 \mathrm{H}), 7.65(\mathrm{t}, \\
J=8,1 \mathrm{H}), 7.47(\mathrm{t}, J=8,1 \mathrm{H})\end{array}$ & $5.97(\mathrm{~s})$ & $\begin{array}{ll}3.60(\mathrm{t}, \quad J=7, & 2 \mathrm{H}), \\
1.54(\mathrm{~m}, & 2 \mathrm{H}), \\
1.24(\mathrm{~m}, 6 \mathrm{H}), & 0.84(\mathrm{t}, \\
J=7,3 \mathrm{H}) & \end{array}$ \\
\hline $5 \mathbf{b}$ & $\begin{array}{l}10.78 \\
(\mathrm{t}, J=6)\end{array}$ & $\begin{array}{l}9.59(\mathrm{~s}, \quad 1 \mathrm{H}), \quad 9.29(\mathrm{~d}, \\
J=6,1 \mathrm{H}), 9.15(\mathrm{~d}, J=7 \\
1 \mathrm{H}), 8.37(\mathrm{t}, J=7,1 \mathrm{H})\end{array}$ & $\begin{array}{l}6.33 \quad(\mathrm{~d}, \\
J=6)\end{array}$ & $\begin{array}{l}8.15(\mathrm{~d}, J=8,1 \mathrm{H}), 8.08(\mathrm{~d}, \\
J=8,1 \mathrm{H}), 7.64(\mathrm{t}, J=8,1 \mathrm{H}) \\
7.46(\mathrm{t}, J=8,1 \mathrm{H})\end{array}$ & $5.98(\mathrm{~s})$ & $\begin{array}{ll}3.60(\mathrm{t}, \quad J=7, & 2 \mathrm{H}), \\
1.54(\mathrm{~m}, & 2 \mathrm{H}), \\
1.24(\mathrm{~m}, 8 \mathrm{H}), & 0.86(\mathrm{t}, \\
J=7,3 \mathrm{H}) & \end{array}$ \\
\hline
\end{tabular}


Table 9. Continued

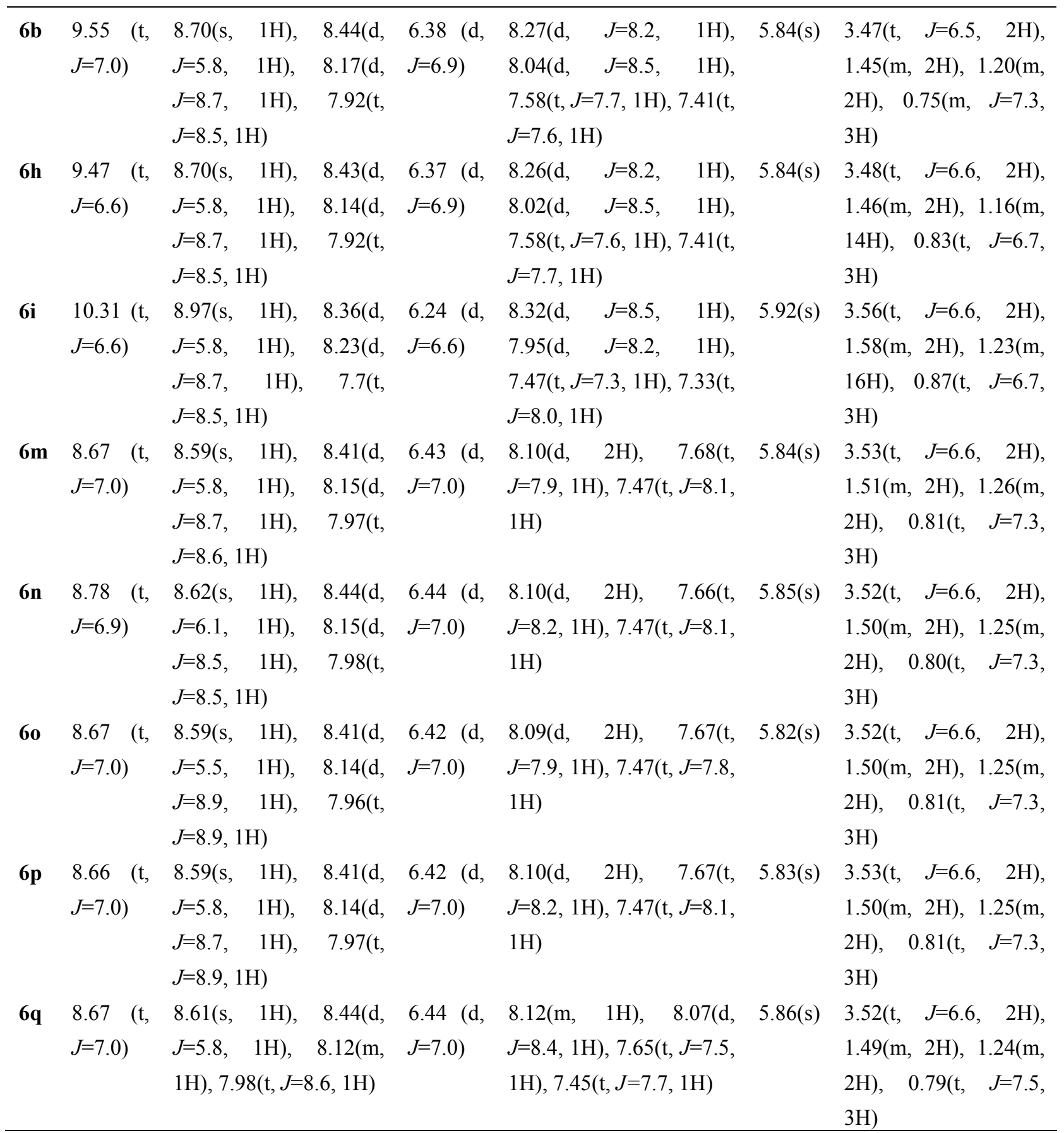

${ }^{\text {a }}$ Solvent: DMSO- $d_{6}$ for $\mathbf{5 a}, \mathbf{5 b}, \mathbf{6 b}, \mathbf{6 m - 6 q}$ and in $\mathrm{CDCl}_{3}$ for $\mathbf{6 i}, \mathbf{6 h}$ 
Table 10. ${ }^{13} \mathrm{C}$ NMR Spectral Data ${ }^{\mathrm{a}}$ of Pyridinium Salts 5,6

\begin{tabular}{|c|c|c|c|c|c|c|}
\hline Salt & $\mathrm{C}=\mathrm{O}$ & Pyridine & $\mathrm{NCH}_{2} \mathrm{~N}$ & Benzotriazole & $\mathrm{NCH}_{2} \mathrm{O}$ & $\mathrm{R}$ \\
\hline $5 a$ & 162.0 & $\begin{array}{l}144.9, \quad 143.3, \\
127.9,127.4,124.1\end{array}$ & 51.4 & $\begin{array}{l}145.1, \quad 145.0, \quad 132.2 \\
132.0,118.9,111.2\end{array}$ & 88.8 & $\begin{array}{l}70.4,30.8,28.6,24.8,21.6 \\
13.9\end{array}$ \\
\hline $5 \mathbf{b}$ & 162.0 & $\begin{array}{l}145.2, \quad 143.6, \\
128.0,127.7,124.3\end{array}$ & 51.4 & $\begin{array}{l}145.3, \quad 145.1, \quad 132.4, \\
132.3,119.1,111.2\end{array}$ & 88.9 & $\begin{array}{l}70.4,31.2,28.6,25.2,22.1 \\
14.0\end{array}$ \\
\hline $6 b$ & - & $\begin{array}{lr}145.5, & 132.1, \\
128.6,128.0,126.7\end{array}$ & 54.8 & $\begin{array}{l}146.2, \quad 132.3, \quad 127.7 \\
124.5,119.3,111.5\end{array}$ & 88.6 & $69.9,30.6,18.3,13.4$ \\
\hline $6 h$ & - & $\begin{array}{lr}145.2, & 131.8, \\
128.4, & 127.7,126.4\end{array}$ & 54.7 & $\begin{array}{l}145.8, \quad 132.0, \quad 127.4, \\
124.2,119.0,111.2\end{array}$ & 88.5 & $\begin{array}{l}70.2,31.3,28.9,28.7,28.6 \\
25.2,22.2,14.0\end{array}$ \\
\hline $6 \mathbf{i}$ & - & $\begin{array}{l}146.1, \quad 129.7, \\
128.5,128.1,126.7\end{array}$ & 55.1 & $\begin{array}{l}147.1, \quad 132.2, \quad 127.7 \\
124.4,119.3,111.1\end{array}$ & 89.3 & $\begin{array}{l}71.8,31.6,29.3,29.2,29.2 \\
29.0,28.9,28.8,25.4,22.3 \\
13.8\end{array}$ \\
\hline $6 \mathrm{~m}$ & - & $\begin{array}{l}145.5, \quad 132.3, \\
128.9,128.1,126.8\end{array}$ & 54.8 & $\begin{array}{l}146.1, \quad 132.3, \quad 127.9, \\
124.6,119.4,111.0\end{array}$ & 88.8 & $70.0,30.6,18.3,13.4$ \\
\hline $6 n$ & - & $\begin{array}{l}145.5, \quad 132.3, \\
128.9,128.1,126.8\end{array}$ & 54.8 & $\begin{array}{l}146.1, \quad 132.3, \quad 127.9 \\
124.5,119.4,111.0\end{array}$ & 88.8 & $69.8,30.6,18.3,13.4$ \\
\hline 60 & - & $\begin{array}{l}145.5, \quad 132.3, \\
128.9,128.1,126.8\end{array}$ & 54.8 & $\begin{array}{l}146.1, \quad 132.2, \quad 127.9, \\
124.6,119.4,111.0\end{array}$ & 88.8 & $70.0,30.6,18.4,13.4$ \\
\hline $6 p$ & - & $\begin{array}{l}145.5, \quad 132.3, \\
128.9,128.1,126.8\end{array}$ & 54.8 & $\begin{array}{l}146.1, \quad 132.3, \quad 127.9 \\
124.6,119.4,111.0\end{array}$ & 88.8 & $70.0,30.6,18.4,13.4$ \\
\hline $6 \mathbf{q}$ & - & $\begin{array}{lr}145.2, & 132.0, \\
128.7,127.8, & 126.5\end{array}$ & 54.8 & $\begin{array}{l}145.7, \quad 132.0, \quad 127.6 \\
124.3,119.1,110.9\end{array}$ & 88.5 & $69.9,30.5,18.3,13.4$ \\
\hline
\end{tabular}

${ }^{\mathrm{a}}$ Solvent: $\mathrm{DMSO}-d_{6}$ for $\mathbf{5 a}, \mathbf{5 b}, \mathbf{6 b}, \mathbf{6 m - 6 \mathbf { q }}$ and $\mathrm{CDCl}_{3}$ for $\mathbf{6} \mathbf{i}, \mathbf{6 h}$

\section{Acknowledgements}

This investigation received financial support from the Polish Committee of Scientific Research Grant KBN No 3 T09B 01015

\section{References}

1. Anthoni, U.; Christophersen, C.; Hongaard, L.; Nielsen, P. H. Comp. Biochem. Physiol. 1991, 99B, 1 .

2. Zhou, J.; Hu, Y; Hu, H. Synthesis 1999, 166.

3. Eicher-Lorka, O.; Kupetis, G. K.; Rastenyte, L.; Matijoska, A. Synthesis 1999, 2131.

4. Scriven, E. F. V. Chem. Soc. Rev. 1983, 12, 129. 
5. Dehmlow, E. V.; Dehmlow, S. S. Phase Transfer Catalysis; $3^{\text {rd }}$ Ed., VCH: Weinheim, New York, Basel, Cambridge, Tokyo, 1993.

6. Maeda, T.; Manabe, Y.; Yamamoto, M.; Yoshida, M.; Okazaki, K.; Nagamune, H.; Kourai, H. Chem. Pharm. Bull. 1999, 47, 1020.

7. Śliwa W. N-Substituted Salts of Pyridine and Related Compounds, Synthesis, Properties, Applications Academic Press: Częstochowa, Poland, 1996.

8. Welton, T. Chem. Rev. 1999, 99, 2071.

9. Salomaa, P. Acta Chem. Scand. 1957, 11, 468.

10. Pernak, J.; Walerowicz, W. Polish J. Chem. 1981, 55, 1109.

11. Pernak, J. Polish J. Chem. 1985, 59, 439.

12. Milata, V.; Kada, R. Collect. Czech. Chem. Commun. 1994, 59, 725.

13. Pernak, J.; Weglewski, J.; Szymańska D. Polish J. Chem. 1996, 70, 1135.

14. Katritzky, A. R.; Lan, L.; Yang, J. Z.; Denisko, O. V. Chem. Rev. 1998, 98, 409.

15. Katritzky, A. R.; Qiu, G.; Yang, B. Synthesis 1998, 704.

16. Pernak, J.; Mrówczyński, B.; Weglewski, J. Synthesis 1994, 1415.

17. Bedford, C. D.; Harris, R. N.; Howd, R. A.; Goff, D. A.; Koolpe, G. A.; Petesch, M.; Miller, A.; Nolen, H. W. III; Musallam, H. A.; Pick, R. O.; Jones, D. E.; Koplovitz, I.; Sultan, W. E. J. Med. Chem. 1989, 32, 493.

18. Bonhote, P.; Dias, A-P.; Papageorgion, N.; Kalyanasundaram, K.; Gratzel, M. Inorg. Chem. 1996, 35, 1168 